\title{
A First Step in the Quest for the Active Constituents in Filipendula ulmaria (Meadowsweet): Comprehensive Phytochemical Identification by Liquid Chromatography Coupled to Quadrupole-Orbitrap Mass Spectrometry
}

\author{
Authors \\ Sebastiaan Bijttebier ${ }^{1}$, Anastasia Van der Auwera ${ }^{1}$, Stefan Voorspoels ${ }^{2}$, Bart Noten ${ }^{2}$, Nina Hermans ${ }^{1}$, Luc Pieters ${ }^{1}$, \\ Sandra Apers \\ Affiliations \\ ${ }^{1}$ University of Antwerp, Natural Products \& Food Research and Analysis (NatuRA), Antwerp, Belgium
2 Flemish Institute for Technological Research (VITO), Business Unit Separation and Conversion Technology (SCT),
Mol, Belgium
}

\author{
Key words \\ - Filipendula ulmaria \\ - Rosaceae \\ - meadowsweet \\ - LC-PDA-amMS \\ - flavonoids \\ - salicylic acid derivatives \\ - tannins
}

\begin{abstract}
$\nabla$

Filipendula ulmaria (meadowsweet) is traditionally used for the treatment of inflammatory diseases and as a diuretic and antirheumatic. Extracts of Filipendulae herba are on the market in the European Union as food supplements. Nevertheless, its active constituents remain to be revealed. During this study, the phytochemical composition of Filipendulae Ulmariae Herba was comprehensively characterised for the first time with two complementary generic ultrahigh-performance liquid chromatography-photodiode ar-
\end{abstract}

\section{Introduction}

\section{$\nabla$}

Preparations from the herb and/or flowers of Filipendula ulmaria (L.) Maxim (Rosaceae) (meadowsweet) have been used traditionally since the late 16th and 17th century for the treatment of inflammatory diseases and as a diuretic and antirheumatic. F. ulmaria is administered as herbal tea, as a powdered herbal substance in solid dosage form for oral use, and as a tincture. In some countries of the European Union, tinctures or possible tincture-based products containing alcoholic extracts of Filipendulae Herba are on the market as food supplements used for complaints such as rheumatic and arthritic pain [1]. Because of its anti-inflammatory properties, Harbourne et al. studied the phenolic content of aqueous F. ulmaria extracts for their incorporation into a beverage as a functional ingredient [2].

It has been shown that meadowsweet contains phenolic constituents such as flavonoid aglycons (e.g., quercetin, kaempferol), glycosylated flavonoids (e.g., rutin, hyperoside, quercitrin, avicularin, astragalin), and hydrolysable tannins (tellimagrandin I and II, rugosin A, B, D, and E) as well as salicylates (salicylic acid, methyl salicylate, salicylaldehyde, salicylalcohol and their glycosides) ray-accurate mass mass spectrometry methods. Selective ion fragmentation experiments with a hybrid quadrupole-orbital trap mass spectrometer significantly contributed to compound identification: a total of 119 compounds were tentatively identified, 69 new to F. ulmaria. A rich diversity of phenolic constituents was detected and only a few non-phenolic phytochemicals were observed. Metabolisation and pharmacological studies should be conducted to investigate which of these constituents or metabolites there of contribute to the activity of $F$. ulmaria after oral intake.
[3-9]. Only a limited number of non-phenolic constituents such as phytosterols, carotenoids, triterpenes, and chlorophyll derivatives have been reported $[7,10,11]$. In view of the phenolic nature of the main constituents reported in meadowsweet, extensive metabolisation after oral intake before absorption can be expected [12]. Indeed, natural products are often prodrugs, e.g., glycosides, which must undergo in vivo metabolic conversion (activation). Salicylic acid, the in vivo metabolite of salicylic alcohol derivatives present in the plant extract, is responsible for part of the pharmacological activity $[5,13]$. Nevertheless, the chemistry of meadowsweet has not been studied in a comprehensive manner and its active constituents remain to be revealed. Phytochemical studies have often dedicated their efforts towards the identification of a limited number of metabolites, which renders the available information fragmented. Usually detectors designed for targeted analysis such as photodiode array detectors (PDA) and triple quadrupole mass spectrometry detectors are used $[2-5,7-9,14]$. These detectors often do not provide sufficient structural information for compound identification in complex mixtures without the use of analytical standards [15]. Due to the lack of commer- 
cially available analytical standards, identification of unknown metabolites, therefore, often result in tedious multistep purification protocols involving solvent extractions followed by sequential fractionations with open-bed liquid chromatography (LC) and/or (semi) preparative high-performance LC (HPLC) $[6,16]$. To obtain unambiguous structure elucidation and compound identification, nuclear magnetic resonance (NMR) still remains indispensable. NMR is, however, not a very sensitive technique and requires compound purification before detection to allow proper structure elucidation. One of the possible strategies to circumvent this sensitivity issue is the use of a solid-phase extraction (SPE) interface in an LC-SPE-NMR configuration [17].

In the past decade, the usage of analytical instrumentation has shifted from simple analytical instrumentation, such as HPLCPDA, towards more selective and more complex separation and detection systems, such as ultrahigh-performance liquid chromatography-photodiode array-accurate mass mass spectrometry (UHPLC-PDA-amMS), to achieve more definite compound identification [18]. Accurate mass MS detectors facilitate the tentative identification of unknown metabolites without the use of analytical standards. Orbitrap MS detectors can routinely generate mass spectra with a resolving power up to 140000 at full width at half maximum (FWHM) and obtain mass accuracies within 1$2 \mathrm{ppm}$; this enables the calculation of the most probable molecular formulae of the generated precursor and product ions [19]. This utility combined with the selectivity and sensitivity of current hyphenated UHPLC-PDA-amMS systems has paved the way towards generic phytochemical analysis and has led to a very significant impact of MS-based technologies in the field of metabolomics [18]. However, a wide diversity of phytochemical structures is produced in nature. Consequently, the complete metabolite profile cannot be extracted with one solvent nor be analysed with one analytical method. Comprehensive phytochemical characterisation should, therefore, be performed with several analytical methods to (ideally) cover the whole range of plant metabolites present in plants.

The goal of the current study was to explore the phytochemical composition of Filipendulae Ulmariae Herba in a comprehensive manner. A generic characterisation platform consisting of two UHPLC-PDA-amMS methods, complementary in terms of polarity, was used to analyse the broad spectrum of phytochemicals present in Filipendulae Ulmariae Herba. This is a first step in the search for the active constituents of $F$. ulmaria.

\section{Results and Discussion}

$\nabla$

Two generic LC-PDA-amMS methods were previously designed to be complementary in terms of polarity: one method for moderately polar compounds such as phenolic constituents [20] and another for apolar phytochemicals such as among others carotenoids and phytosterols [21]. The current study is the first to combine both methods into a platform for comprehensive phytochemical characterisation. A hybrid quadrupole-orbital trap MS analyser (Q Exactive ${ }^{\mathrm{TM}}$, Thermo Fisher Scientific) was used, which enables selective ion fragmentation, a functionality that contributes significantly to compound identification by generating clean product ion spectra. Selective ion fragmentation is particularly useful for associating product ions with precursor ions during coelution of multiple compounds, as is often the case in complex plant extracts.
Although data-dependent fragmentation $\left(\mathrm{ddMS}^{2}\right)$ provides vast amounts of structural information, in some cases, the generated product ions may not suffice for full characterisation of the substructures (e.g., aglycon moieties of flavonoid glycosides). In these cases, in-source collision-induced dissociation (CID) fragmentation was used to generate substructure product ions in a first step. These in-source product ions were subsequently selected with the quadrupole for higher energy collisional dissociation (HCD) fragmentation to obtain pseudo $\mathrm{MS}^{3}$ spectra for their tentative identification.

Structures were assigned to unknown peaks only when both the mass/charge $(\mathrm{m} / \mathrm{z})$ ratios and molecular formulae of the precursor and product ions were in agreement. PDA spectra and retention times often provided additional confirmation of the proposed structures. The information obtained by analysis is, however, not always sufficient for peak identification at an acceptable confidence level. Additional information for successful dereplication was often acquired from in-house and commercial compound databases and peer reviewed publications. Tables 1 and 2 show the diagnostic amMS and PDA data used for chromatographic peak identification. These tables also specify the literature consulted for confirmation of compound identity.

Application of a generic LC-PDA-amMS method for the identification of moderately polar phytochemicals enabled the identification of a multitude of phenolic constituents, many of which have never been reported before in F. ulmaria. Compounds identified for the first time in F. ulmaria are indicated in italics in $\odot$ Tables 1 and 2. Fig. 1 provides an overview of the retention times and $\mathrm{m} / z$ values in the heated electrospray ionisation (HESI) negative mode of the compounds identified with this method. The data labels match the compound numbers in Table 1. Predominantly deprotonated molecules $\left([\mathrm{M}-\mathrm{H}]^{-}\right)$were formed in the negative ionisation mode. Formic acid adducts $\left([\mathrm{M}-\mathrm{H}+\mathrm{FA}]^{-}\right.$), deprotonated dimers $\left([2 \mathrm{M}-\mathrm{H}]^{-}\right)$, doubly deprotonated molecules $\left([\mathrm{M}-2 \mathrm{H}]^{2-}\right)$ and product ions caused by in-source fragmentation were also observed ( Table 1 ). In the positive ionisation mode, predominantly protonated molecules $\left([\mathrm{M}+\mathrm{H}]^{+}\right)$were generated and, to a lesser extent, also sodium adducts, ammonium adducts, doubly protonated molecules $\left([\mathrm{M}+\mathrm{Na}]^{+},[\mathrm{M}+\right.$ $\left.\mathrm{NH}_{4}\right]^{+},[\mathrm{M}+2 \mathrm{H}]^{2+}$, respectively), and product ions caused by insource fragmentation ( $\bullet$ Table $\mathbf{1}$ ).

Flavonoids chiefly occurred as glycoconjugates (with hexose, pentose, deoxyhexose, dihexose, and hexose-deoxyhexose moieties; Table 1). Several of the glycosides were identified with analytical standards. Other flavonoid glycoconjugates were identified with ddMS ${ }^{2}$ : HCD fragmentation resulted in the distinct presence of product ions caused by the loss of sugar moieties, indicating glycosidic O-linkages [22]. Various glycosyl flavonoids acylated with (di)galloyl moieties were also detected. In-source CID fragmentation and subsequent HCD fragmentation of the aglycon moiety (pseudo $\mathrm{MS}^{3}$ ) often allowed for the tentative identification of the flavonoid moieties. Fragmentation of flavonoid aglycons has been reviewed in detail previously, among others by Cuyckens and Claeys [22]. The main flavonoid moieties of the detected glycosides consisted of quercetin and kaempferol, in line with previous studies [6,9]. Multiple flavonoid aglycons were found, such as among others quercetin, kaempferol, catechin, epicatechin, epigallocatechin, apigenin, isorhamnetin, and luteolin. Several flavonoids that were previously reported (e.g., ulmarioside, epigallocatechingallate, and isorhamnetin acetylhexoside) were not detected, which is probably due to natural phytochemical variations in between plants and plant parts ex- 

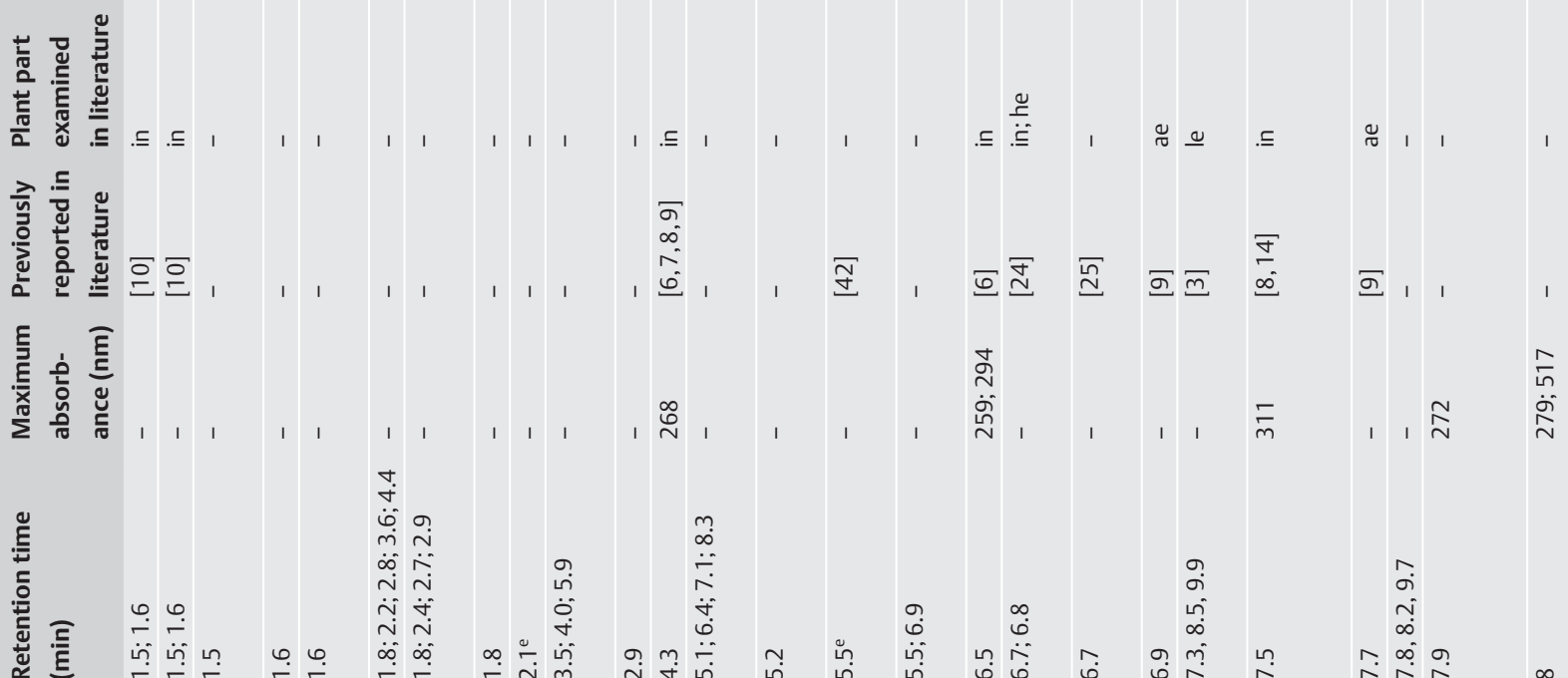

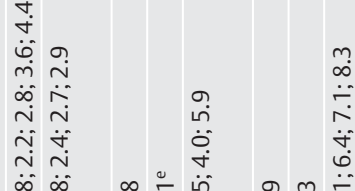

衰它

๘
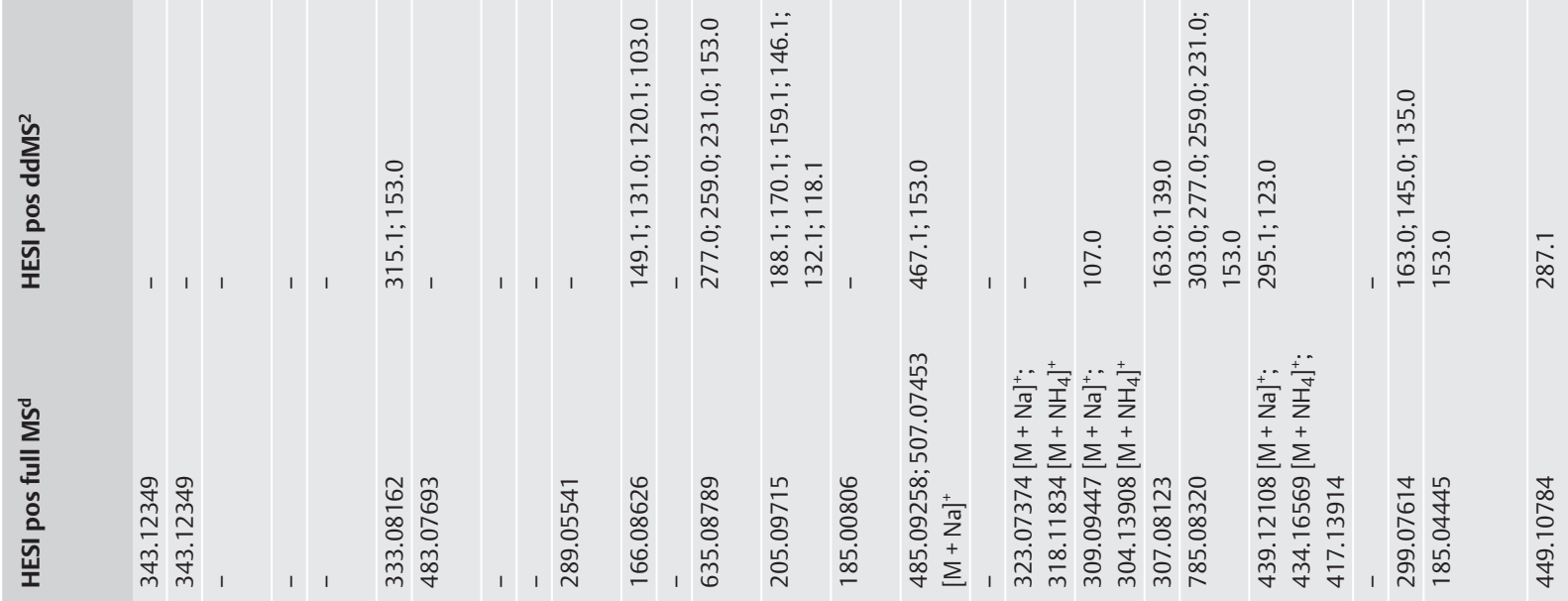

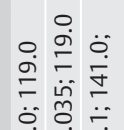

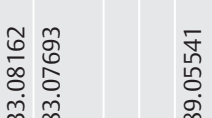
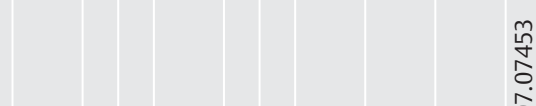

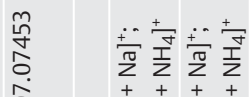

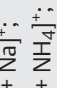

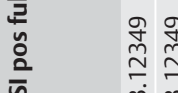

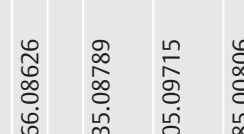

容 $\quad \sum^{+} \sum^{+} \sum^{+} \sum^{+} \sum^{+}$

$\sum^{+} \sum^{+}$

荘 $\quad \dot{m} \frac{m}{m}$

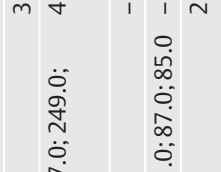

$\stackrel{\circ}{\circ}$,

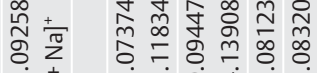

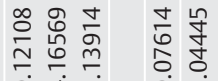

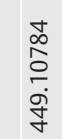

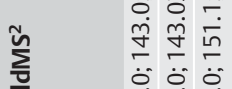

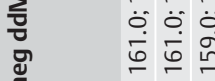

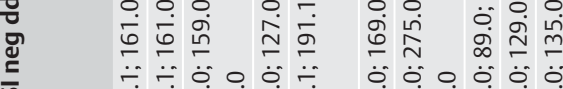

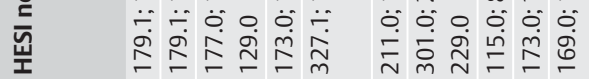

$\underset{\substack{0 \\ \dot{g}}}{\ddot{g}}$

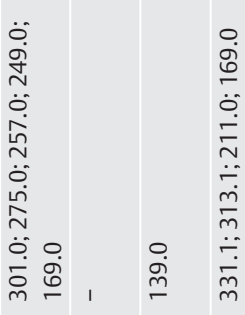

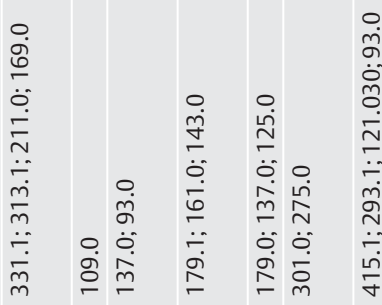

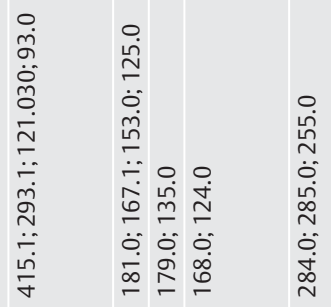

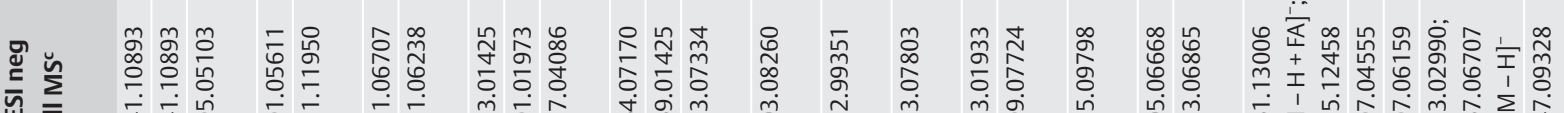

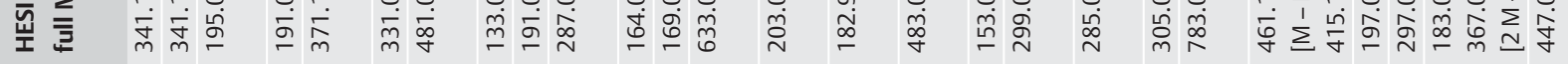

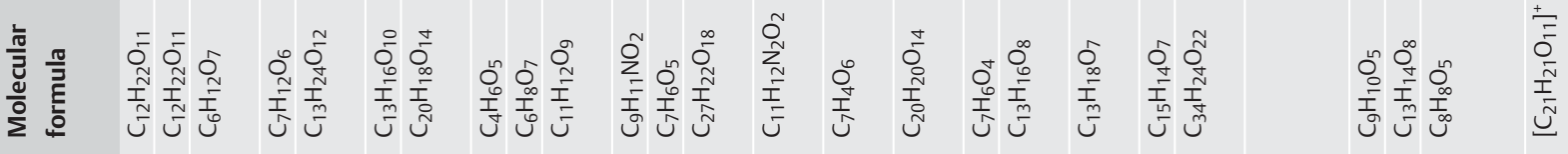
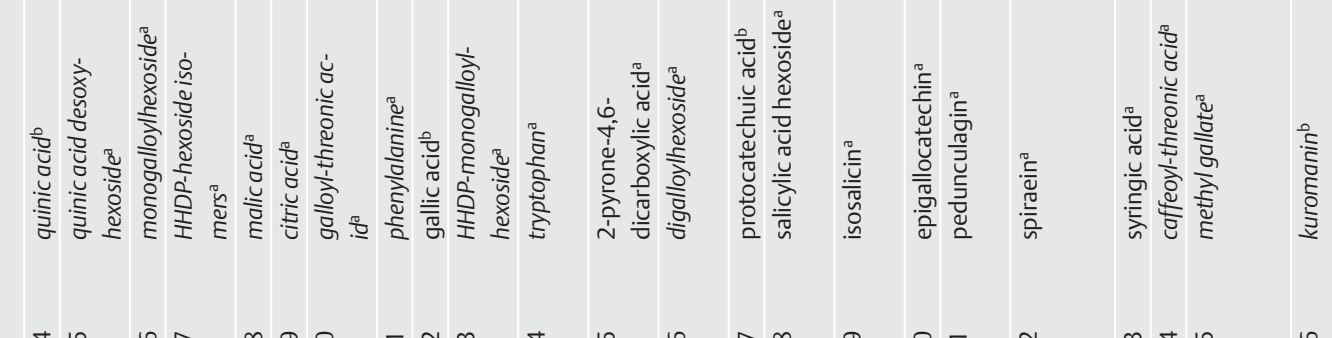

$\cdots=\geq \geq \geq$

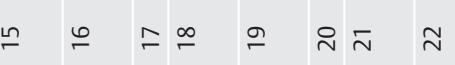

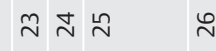




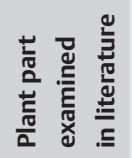

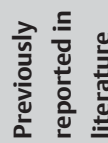

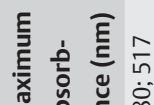

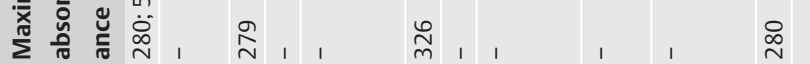
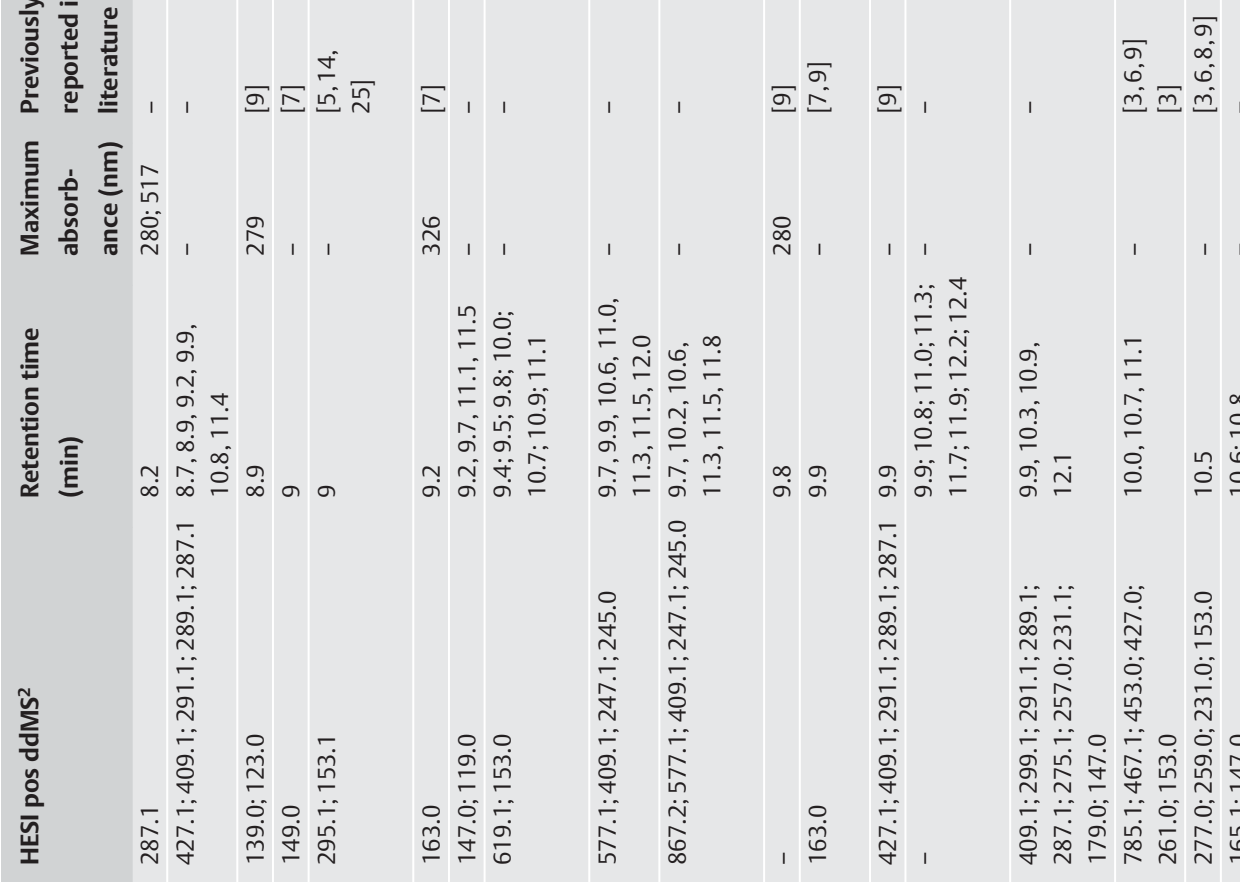

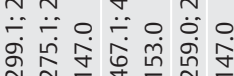

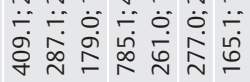

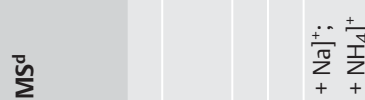

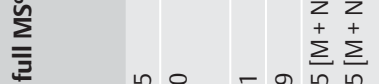

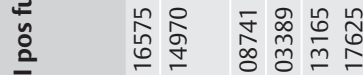

至 岁灾

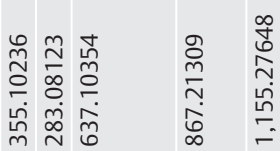

$\underset{\substack{n\\}}{\stackrel{n}{n}}$

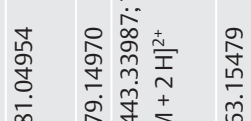

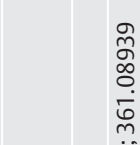

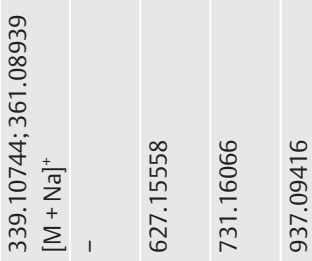

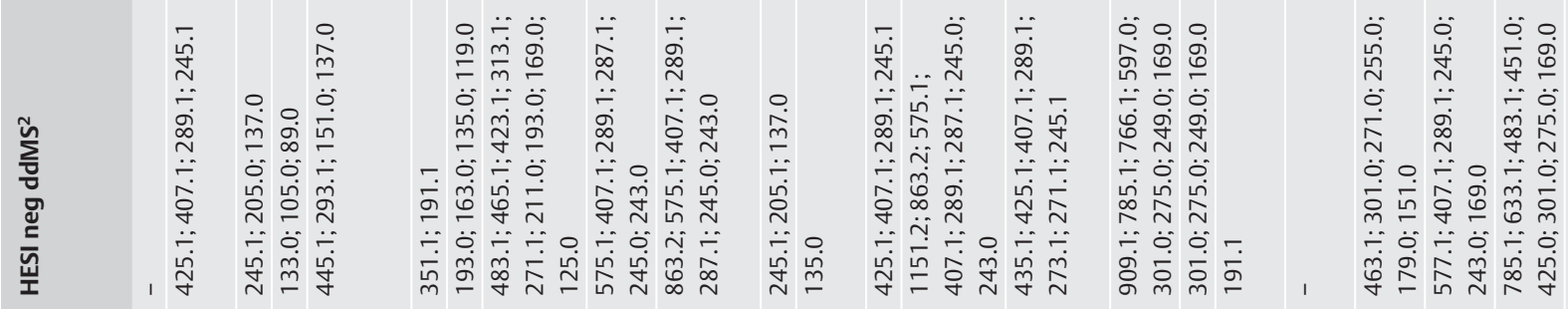

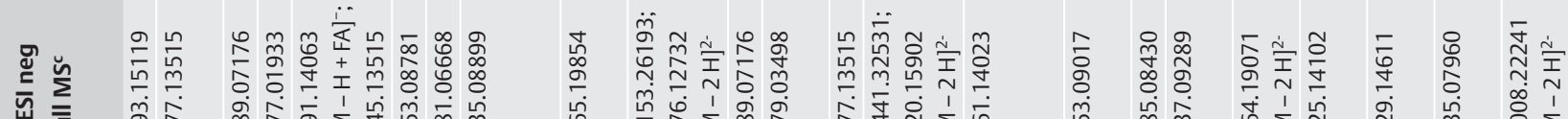

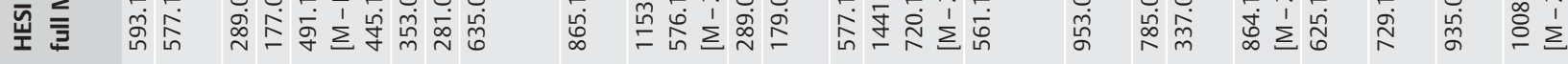

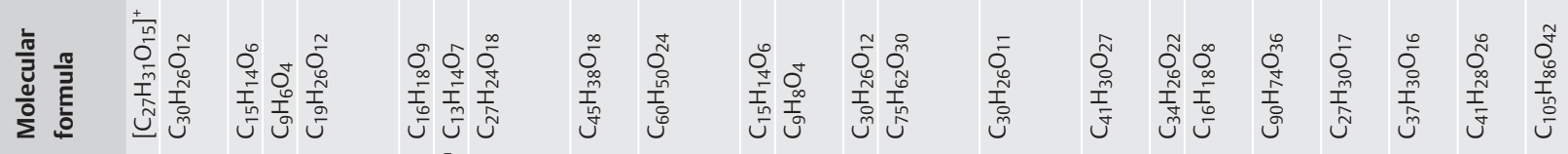

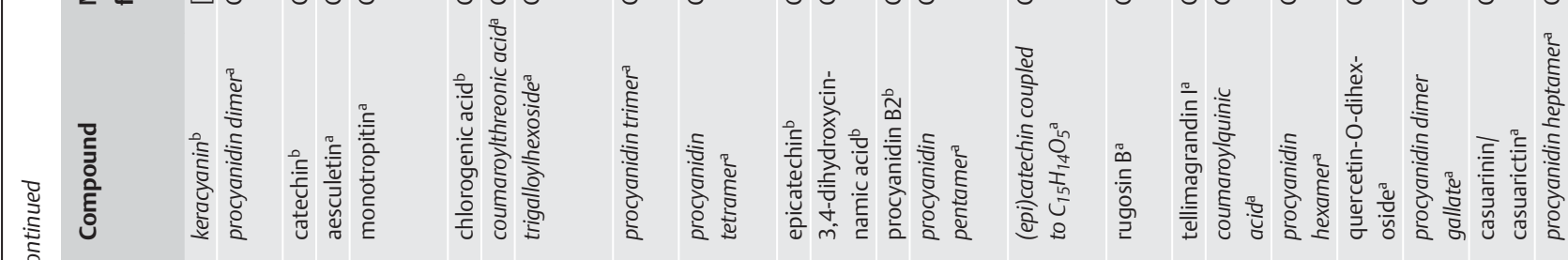
ฮे 

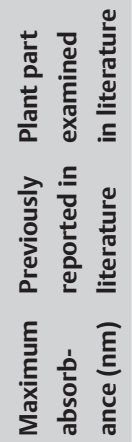

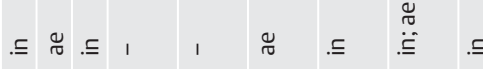

范

$\begin{array}{lll} & & \sigma \\ \sigma & \sigma & \infty \\ 0 & 0 & 0 \\ 0 & 0 & 0\end{array}$

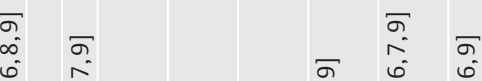

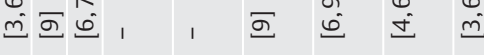

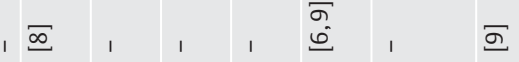

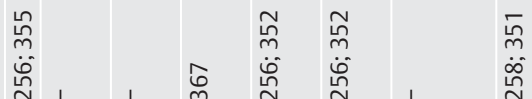

$\stackrel{\substack{m \\ \stackrel{n}{n}}}{\stackrel{n}{n}}$

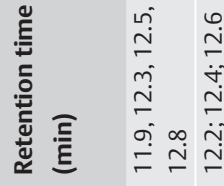

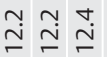

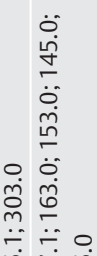

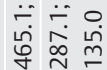

๕ั

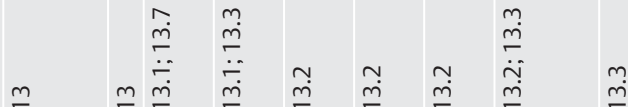

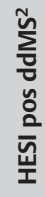

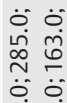

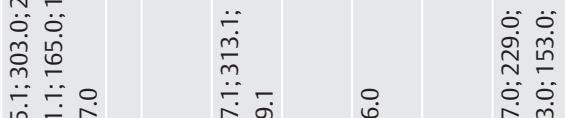

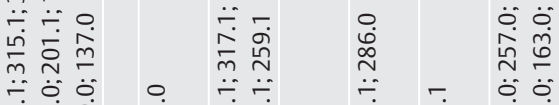

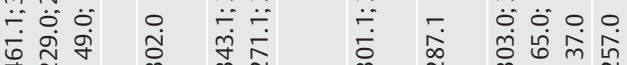

+ i m m

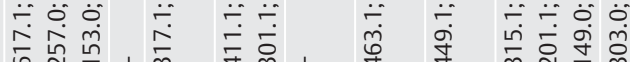

$\frac{\sum^{n}}{3}$

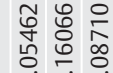

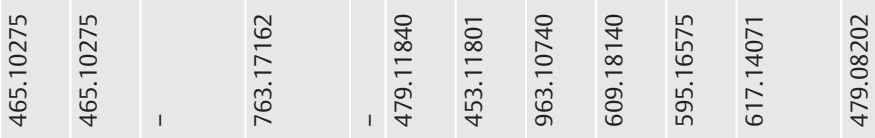

苛

o. 0.00

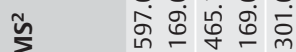

$\because \ddot{\circ} \because \ddot{\circ}$

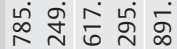

เู่

, 岁 岁

ํ.

نे

물

高 岁家

$\ddot{\circ}$ ö

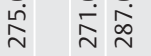

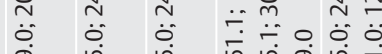

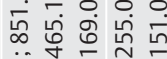

$\ddot{0}$ ö

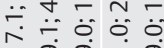

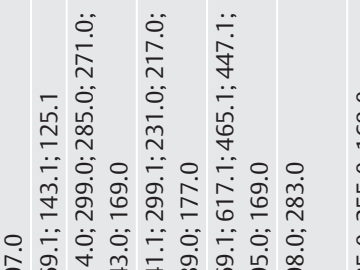

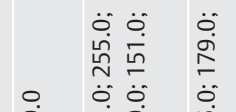

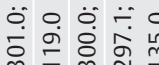

กิ

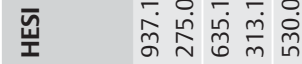

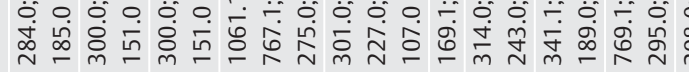

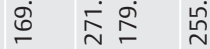

मे

O.

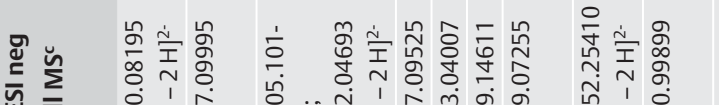

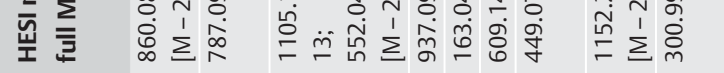

\section{ని}

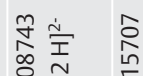

量

$\approx$

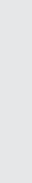

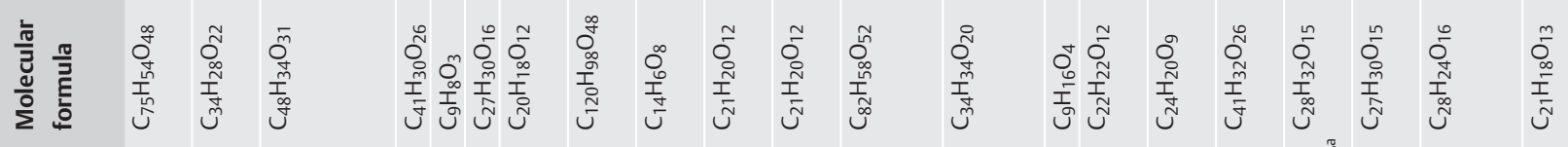

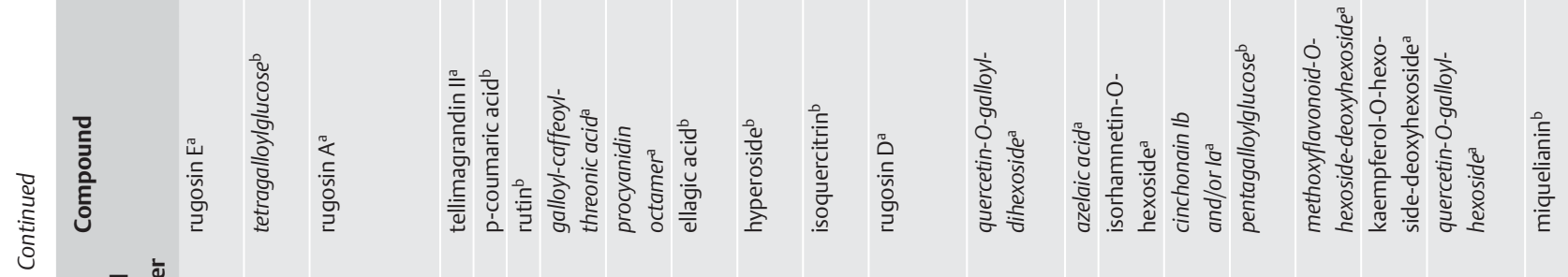

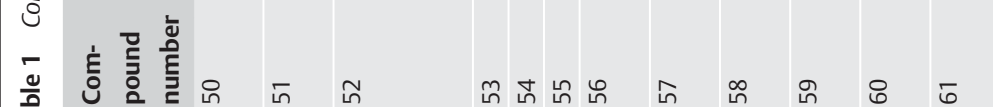




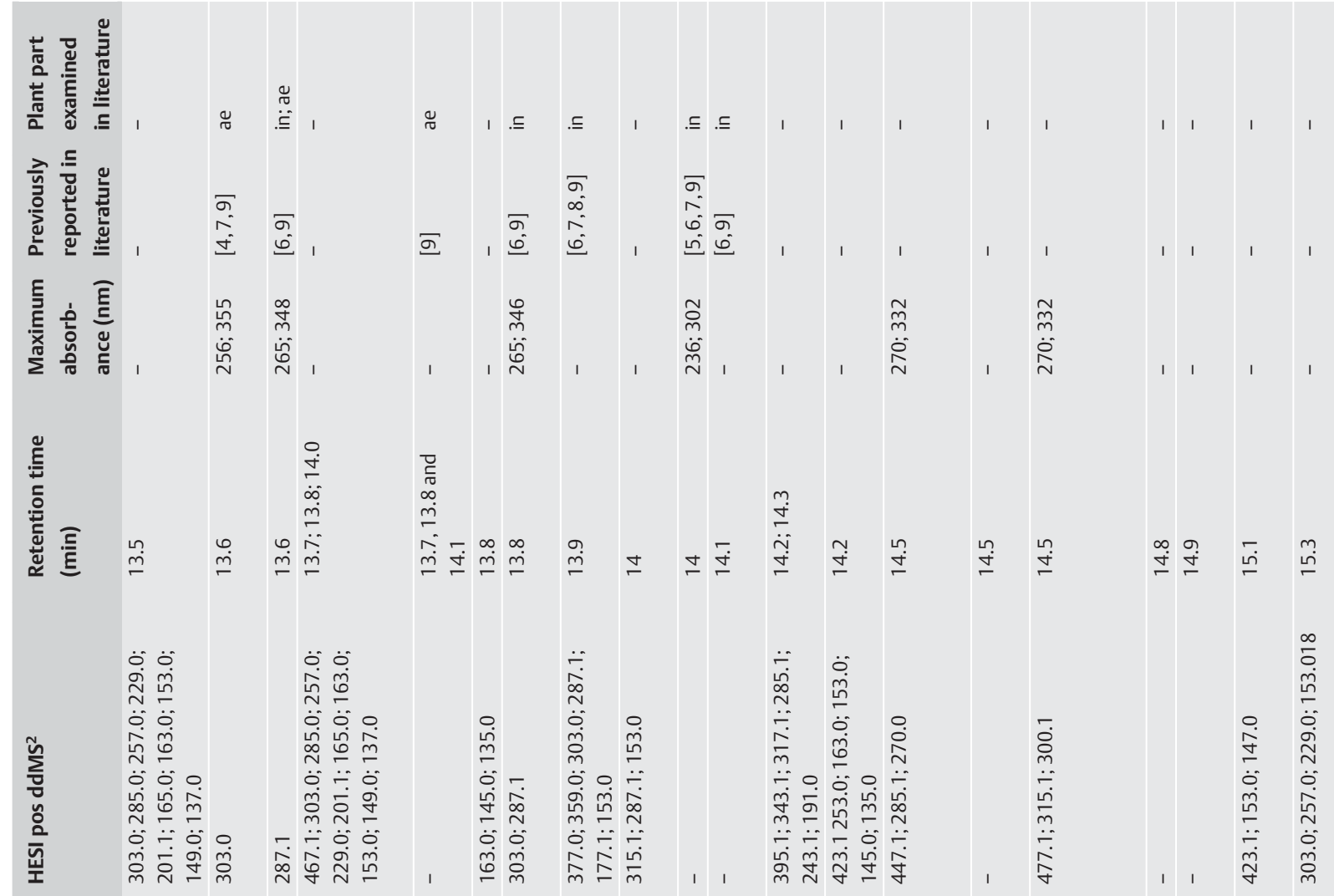

$$
\text { ! }
$$

\section{ent}

\section{II}

高

용

\section{:}

후잉

业

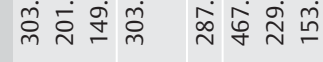



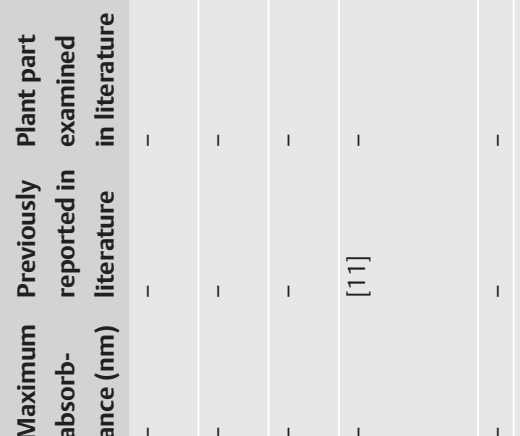

कू

๑.

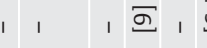

$\Xi$

E

镸震

$\stackrel{\substack{n \\ \stackrel{n}{n}}}{\stackrel{\text { I }}{N}}$

苾

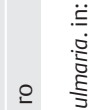

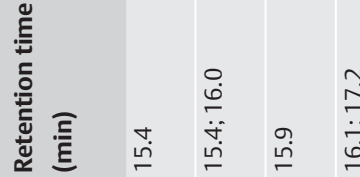

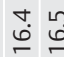

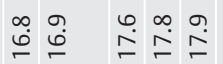

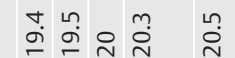

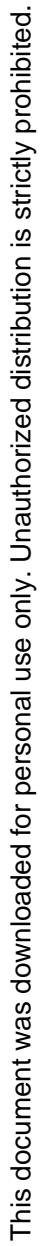

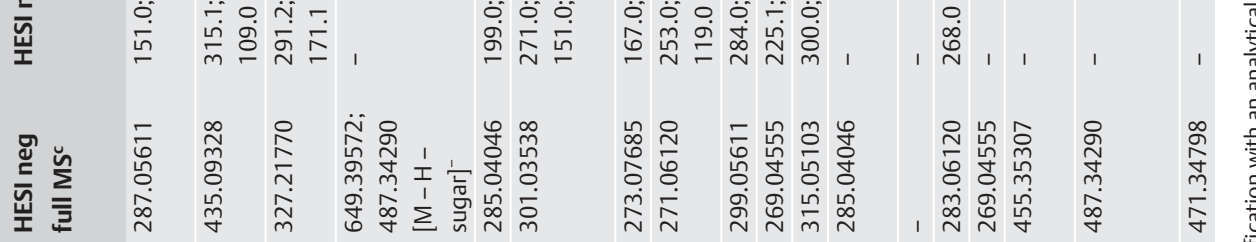

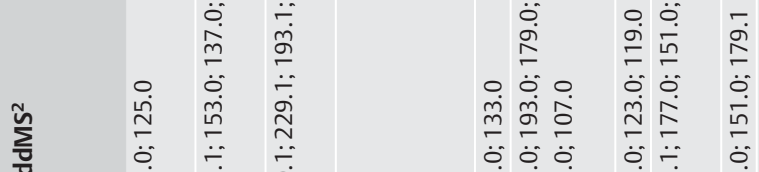

च मिं

I

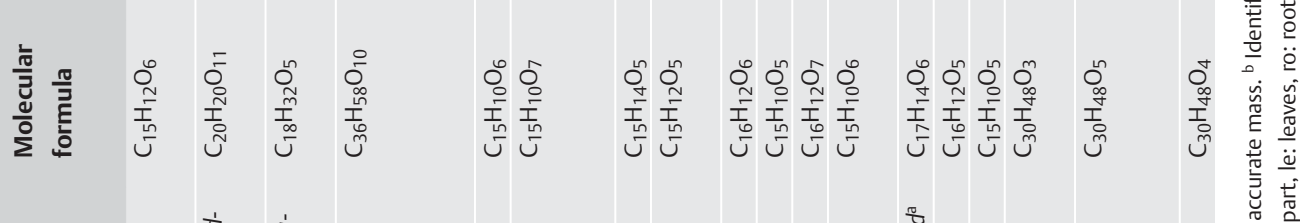

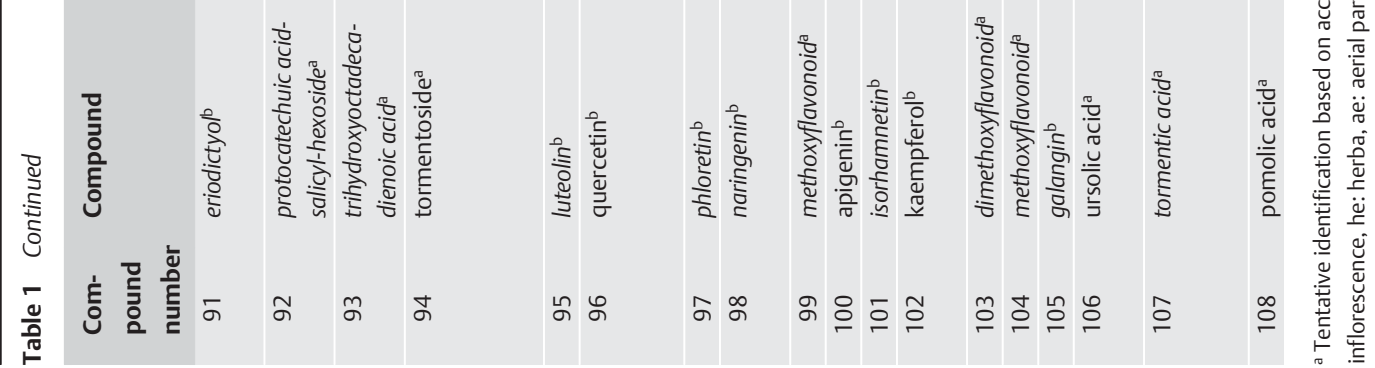




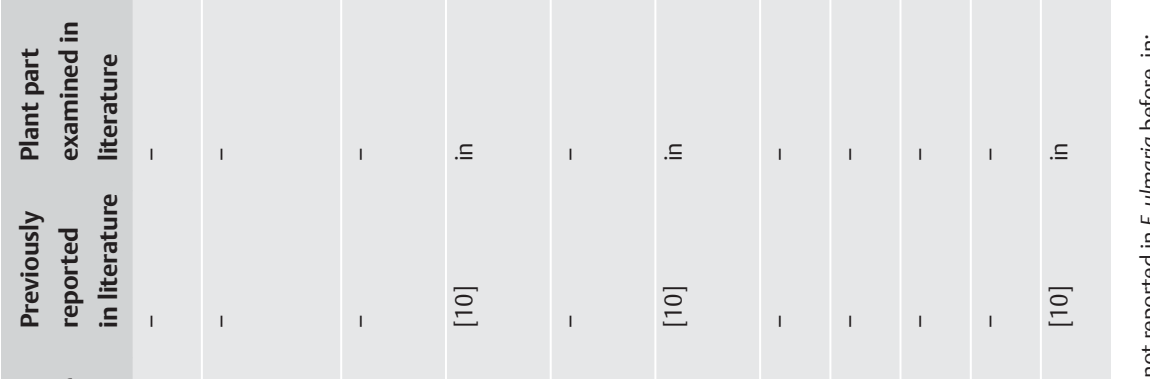

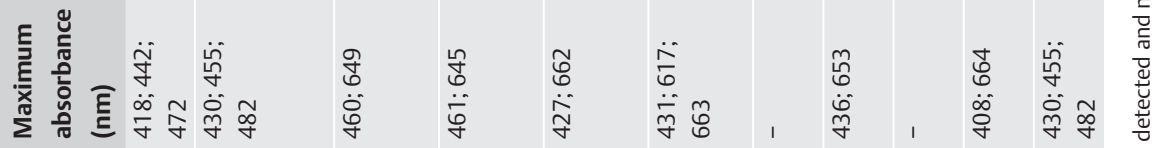

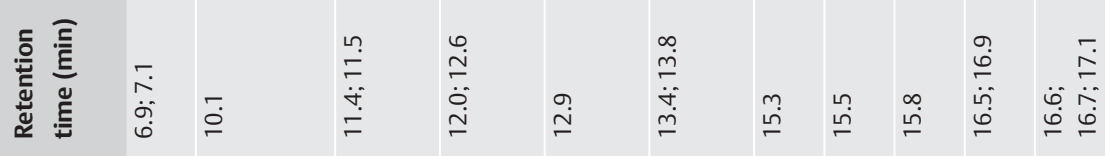

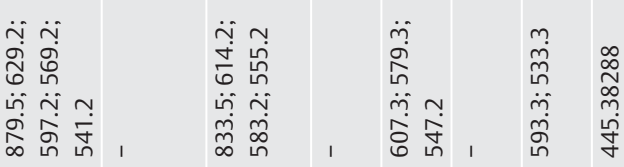

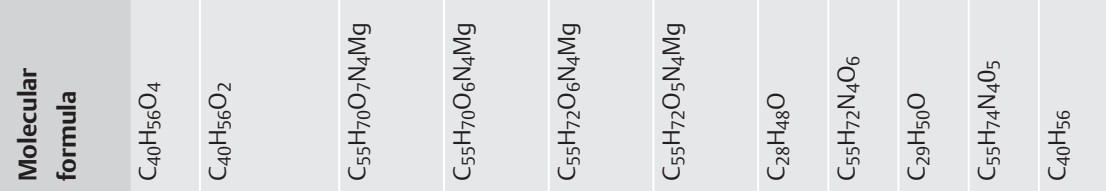

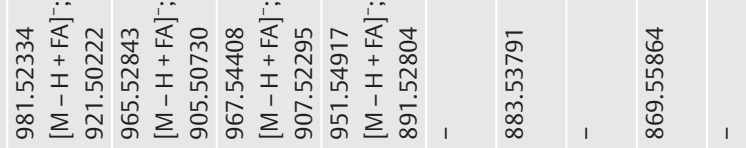

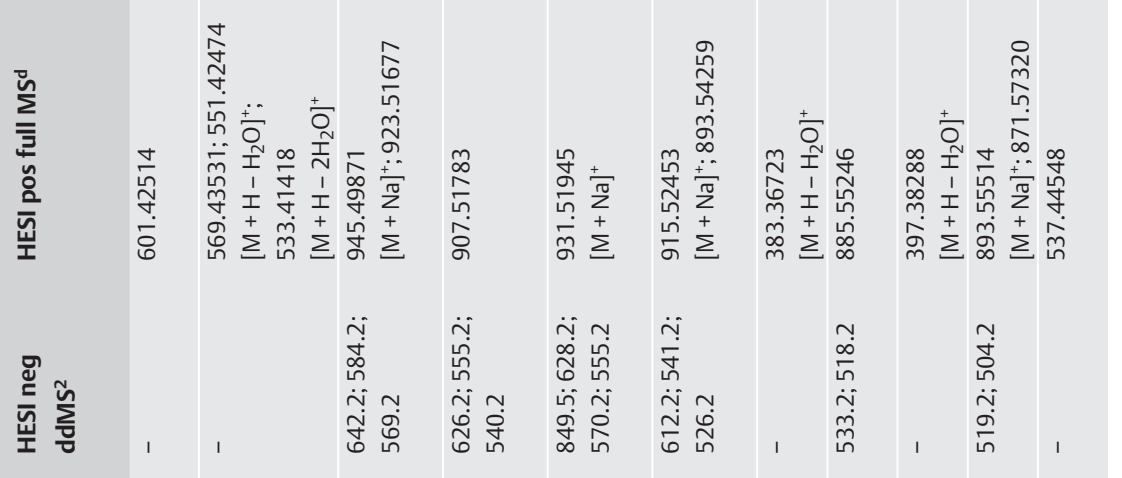

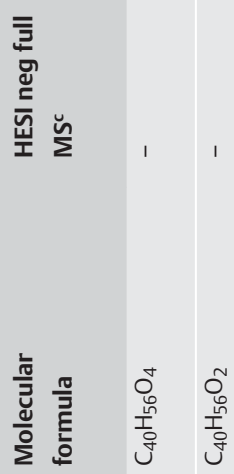

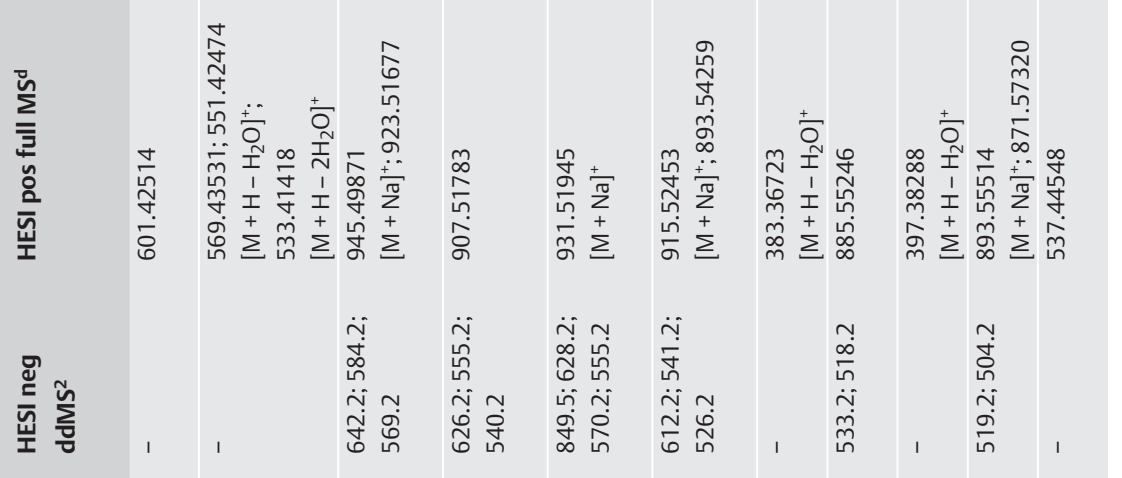

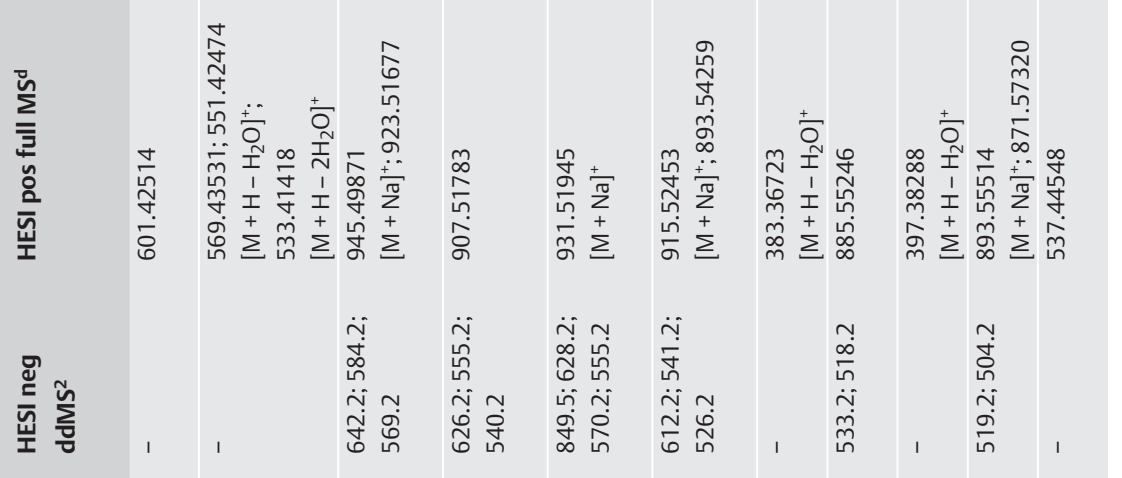

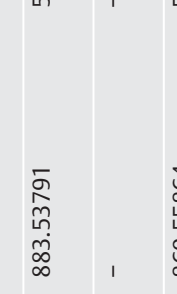

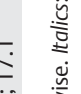




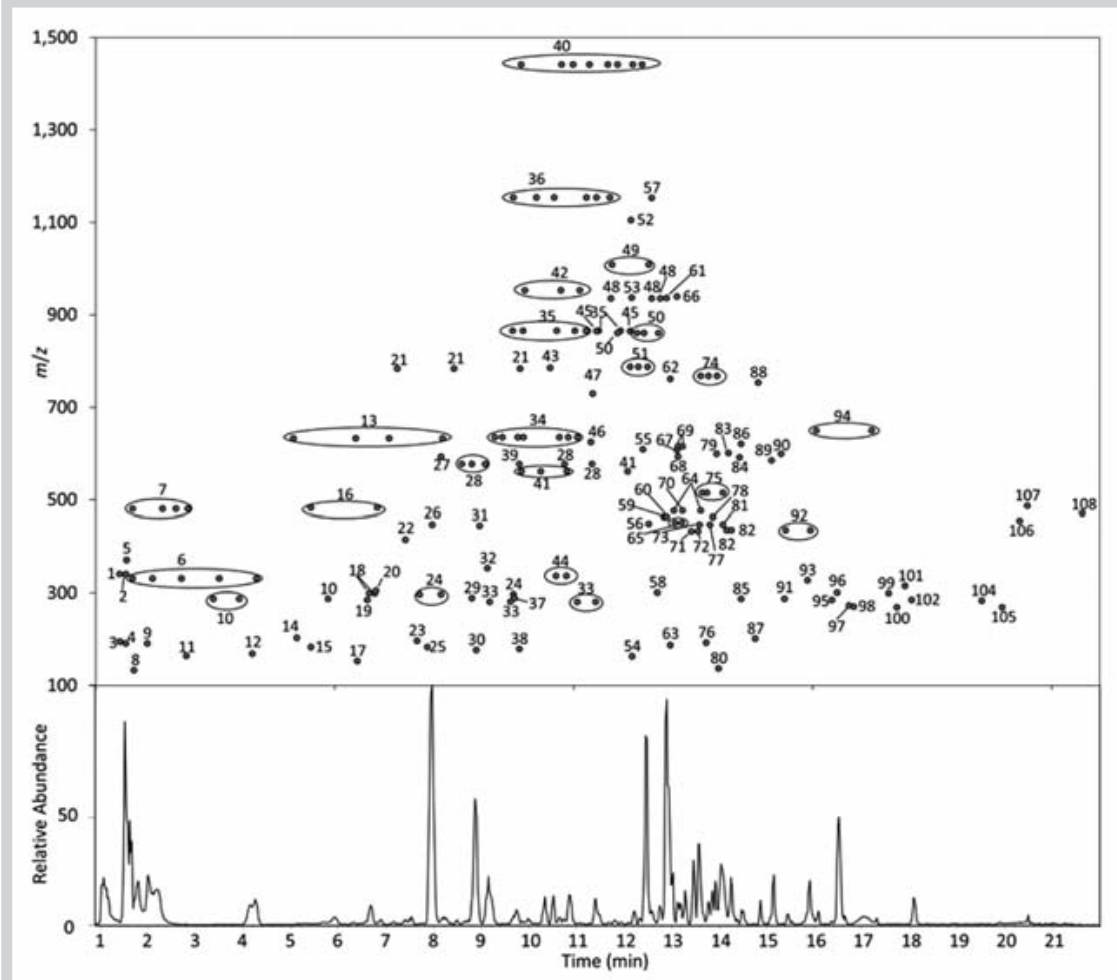

Fig. 1 Plot of the retention times vs. $m / z$ values of the compounds identified in $F$. ulmaria detected with a generic LC-PDA-amMS method for moderately polar phytochemicals. The data labels match the compound numbers in $\odot$ Table 1.

amined $[8,9]$ or due to a difference in method/instrument sensitivity. Nevertheless, various flavonoid aglycons and glycoconjugates, of which several have never been described in F. ulmaria before (indicated in italics in $\odot$ Table 1 ), have been identified during the current study.

Some phenolic acids such as gallic acid, protocatechuic acid, syringic acid and salicylic acid, and hydroxycinnamic acids such as caffeic acid and p-coumaric acid were detected in the free form ( Table 1), in agreement with previous studies [5,9]. All free phenolic and hydroxycinnamic acids except syringic acid were identified with analytical standards. The product ions of syringic acid generated by ddMS ${ }^{2}$ matched with those described by Sun et al. [23]. Glycosylated salicyl derivatives (salicylic acid hexoside, isosalicin, monotropitin, and spiraein) were also present, while methyl salicylate, salicyl alcohol, and salicyl aldehyde, previously reported in F. ulmaria, were not found [5, 14,24,25]. Next to salicylates, a rich diversity of phenolic and hydroxycinnamic acid oligomers was detected, most of which have never been reported before in F. ulmaria (indicated in italics in 0 Table 1). Tentative identification of these oligomers was based on the consecutive loss of phenolic monomer units during ddMS ${ }^{2}$ ( Table 1) [26]. The phenolic oligomers consisted predominantly of structures containing galloyl, caffeoyl, quinoyl, and coumaroyl moieties. Several phenolic acid and hydroxycinnamic acid oligomers were also detected as glycoconjugates. Often multiple chromatographic peaks were found for the same precursor ion, indicating the presence of structural isomers due to different linkage sites and/or different sugar moieties.

Several of the tentatively identified oligomeric phenolic acid structures described in the previous section may be classified as gallotannins (hydrolysable tannins) [27]. Some of these gallotannins are metabolic precursors of ellagitannins in plants (e.g., pentagalloylglucose is a precursors of tellimagrandin II) [26, 28]. F. ulmaria has been described as a rich source of various monomeric ellagitannins such as casuarinin, casuarictin, pedunculagin, tellimagrandins I and II, rugosins A and B. and dimeric ellagitannins rugosins $D$ and $E[3,6,8,9]$. Such hydrolysable tannins consist of one or more hexahydroxydiphenic acid (HHDP) moieties and several galloyl and glucose moieties [3,6]. During the current study, ionisation of dimeric rugosins resulted in significant amounts of $[\mathrm{M}-2 \mathrm{H}]^{2-}$ ions ( Table 1 ). The fragmentation patterns of the ellagitannins were characterised by consecutive losses of monomeric units ( $\bullet$ Table 1 ). Next to previously reported hydrolysable tannins, other ellagitannins were also tentatively identified during this study (indicated in italics in $\bullet$ Table 1); several HHDP-monogalloyl-hexoside and HHDP-hexoside isomers, similar to the structures of tellimagrandins, were detected. Ellagic acid, which may be formed by hydrolysis of ellagitannins and occurs in multiple plants, fruits, and nuts, was also identified during this study using an analytical standard [29].

Only one recent study has investigated the presence of procyanidins in F. ulmaria. Olennikov and Kruglova detected procyanidins B1 and B2 [9]. During the current study, a multitude of procyanidins ranging from dimeric to octameric isomers was tentatively identified. Procyandin dimer gallate was also tentatively identified. The fragmentation pattern of the detected procyanidins obtained with ddMS ${ }^{2}$ corresponded to that of an analytical standard solution of procyanidin B2 and is in accordance with their fragmentation described by Regueiro et al. [26]. Increasing amounts of $[\mathrm{M}-2 \mathrm{H}]^{2-}$ ions were observed with increasing procyanidin mass, starting from tetramers. For hexamers, heptamers, and octamers, only the $[\mathrm{M}-2 \mathrm{H}]^{2-}$ ion was observed. Because of the low abundant $[\mathrm{M}-2 \mathrm{H}]^{2-}$ signals of the procyanidin hexamers, heptamers, and octamers, selective fragmentation could not be used to confirm their identity. Nontheless, the use of a hybrid orbitrap mass analyser enabled the tentative identification of a large amount of tannins that have never been reported before in $F$. $u l$ maria (indicated in italics in $\bullet$ Table 1 ). 


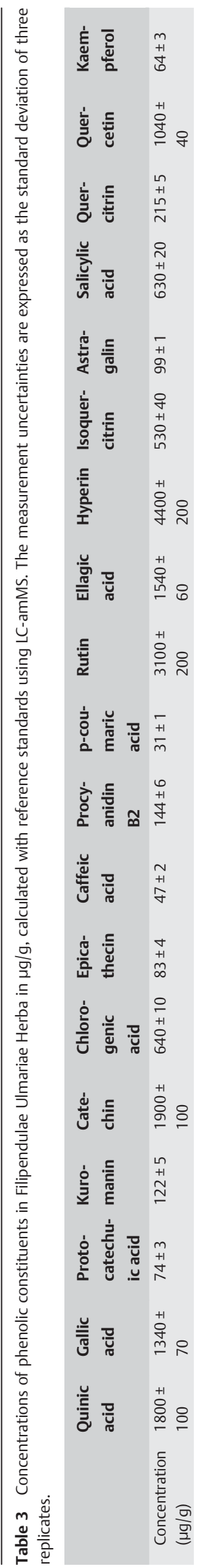

Based on the most abundant signals observed with PDA detection, the main phenolic constituents of Filipendulae Ulmariae Herba were identified: gallic acid (12), procyanidin dimer (28; not B2), rugosin A (52) or tellimagrandin II (53), rutin (55), hyperoside (59), isoquercitrin (60), quercetin-O-galloyldihexoside (62), kaempferol-O-hexoside-deoxyhexoside (68), quercetin-Ogalloylhexoside (69), quercetin-O-pentoside (71), avicularoside (72), astragalin (73), quercetin-O-hexoside (78), digalloyl-caffeoyl-threonic acid (83), methoxyflavonoid-O-hexoside-deoxyhexoside (84), dimethoxyflavonoid-O-hexoside-deoxyhexoside (86), digalloyl-coumaroyl-threonic acid (89), and quercetin (96). These results show that the main constituents predominantly consist of flavonoid glycosides and tannins. Compounds for which a reference standard was available were quantified with LC-amMS ( Table 3). The concentrations detected during this study are in accordance with those found by Fecka, who investigated the concentration of selected polyphenols in dried flowers of F. ulmaria. [6].

Consistent with previous literature, only few and frequently low abundant non-phenolic phytochemicals were detected in Filipendulae Ulmariae Herba. Some triterpenes were tentatively identified with the generic LC-PDA-amMS method for moderately polar phytochemicals, while chlorophyll derivatives, phytosterols, and carotenoids were detected with the complementary LC-PDA-amMS method for apolar phytochemicals. Ionisation in the negative and positive modes during analysis with the LC-PDA-amMS method for apolar phytochemicals predominantly rendered $[\mathrm{M}-\mathrm{H}]^{-}$and $[\mathrm{M}-\mathrm{H}+\mathrm{FA}]^{-}$ions and $[\mathrm{M}+\mathrm{H}]^{+}$and $[\mathrm{M}+$ $\mathrm{Na}]^{+}$ions, respectively. Product ions due to in-source fragmentation (e.g., loss of water and sugar moieties) were also observed (- Table 2).

Ursolic acid, pomolic acid, and tormentoside, a glycoside of tormentic acid, have been described to be present in the roots of F. ulmaria [11]. During this study, these compounds were also tentatively identified based on product ions formed by selective HCD fragmentation in positive ionisation mode ( Table 1). Formation of product ions by retro Diels-Alder fragmentation according to the fragmentation proposed by Li et al. was observed for all triterpenes [30]. Consecutive losses of water and $\mathrm{CO}_{2}$ moieties (and a sugar moiety for tormentoside) were also detected. Two chromatographic peaks were detected for tormentoside, indicating the presence of two isomers. Next to the previously reported triterpenes, tormentic acid was tentatively identified for the first time in F. ulmaria during the current study, with a fragmentation pattern similar to the other triterpene aglycons (- Table 1).

Barros et al. reported the presence of chlorophylls $a$ and $b$ in $F$. ulmaria [10]. These compounds were also tentatively identified based on their MS fragmentation spectra and UV absorbance maxima. Two chromatographic peaks were observed for the respective chlorophylls, corresponding to chlorophyll epimers $a$ and $a^{\prime}$ and $b$ and $b^{\prime}$ [31]. Moreover, other chlorophyll derivatives, such as phaeophytins, hydroxychlorophylls and dihydroxychlorophylls, were also detected, compounds that have not been reported before in F. ulmaria ( Table 2).

Although only present in low amounts, two phytosterols (campesterol and $\beta$-sitosterol) that have not been described previously in F. ulmaria were identified with analytical standards ( Table 2). Both phytosterols were characterised by abundant $\left[\mathrm{M}+\mathrm{H}-\mathrm{H}_{2} \mathrm{O}\right]^{+}$ions due to in-source fragmentation. These main $\left[\mathrm{M}+\mathrm{H}-\mathrm{H}_{2} \mathrm{O}\right]^{+}$ions of both phytosterols appeared at several retention times throughout the chromatogram (data not shown). 
Application of the same LC-PDA-amMS method in a former study has shown that the main in-source produced ion of a free sterol is also the main detected ion of its derivatives, i.e., acylated sterols, steryl glycosides and acylated steryl glycosides, generated by the loss of the attached sugar/fatty acid moieties due to in-source fragmentation [21]. The deprotonated molecules of the derivatives usually are detected in the negative ionisation mode, thereby revealing the identity of the attached sugar and/or fatty acid. Due to their low abundances, they could, however, not be detected during the present study.

Carotenoids have only been scarcely investigated in F. ulmaria; Barros et al. has reported the presence of lycopene and $\beta$-carotene in inflosescences [10]. Lycopene was not detected during the current study. Small amounts of $\beta$-carotene, lutein, and violaxanthin were, however, identified with analytical standards (@ Table 2).

Barros et al. identified tocopherols in inflorescences of F. ulmaria, but they were not detected during this study [10]. Other compounds such as organic acids, amino acids, free sugars, and lipids (data not shown) were tentatively identified with the LC-PDAamMS platform. Although these findings are interesting from an analytical perspective, the biological functions of these compounds are known and no direct contribution to the specific pharmacological activity of $F$. ulmaria is expected.

Salicylic acid, the in vivo metabolite of salicylic alcohol derivatives, is responsible for part of the pharmacological activity of F. ulmaria $[5,13]$. During the current study, several metabolic precursors of salicylic acid were detected. However, a large diversity of other phytochemicals were identified that are likely to contribute as well to the activity of F. ulmaria. In view of the phenolic nature of the main constituents, extensive metabolisation after oral intake before absorption can be expected. It has been estimated that more than $90 \%$ of ingested polyphenols are not absorbed in the small intestine and, thus, remain in the colon at a high concentration where they are extensively metabolised by gut microbiota to produce smaller molecules [32].

In the human gastrointestinal tract, ingested dietary ellagitannins are hydrolysed to release ellagic acid and further metabolised by the colon microbiota to produce dibenzopyranones known as urolithin derivatives (less potent antioxidants as they have lost their free-radical scavenging activity) [27,29]. Urolithins appear in human systemic circulation within a few hours after consumption [29]. Ellagitannin, ellagic acid, and derived metabolites have antioxidant functions, estrogenic and/or antiestrogenic activities, and anti-inflammatory and prebiotic effects [29]. A five-week supplementation of pomegranate juice or pomegranate fruit extract in obese Zucker rats fed an atherogenic diet, for instance, showed a significant decrease in the expression of vascular inflammation markers [33].

Procyanidins have been shown to mediate several anti-inflammatory mechanisms involved in the development of cardiovascular disease [34]. Although there is no consensus on the absorption and metabolism of procyanidins thus far, they seem to be poorly absorbed in the gut due to their polymeric nature and high molecular weight [32]. A possible degradation into flavan-3-ols and low molecular weight products such as phenolic acids and valerolactones can be an explanation for the health effect of procyanidins $[32,35]$. These low molecular weight phenolic compounds can be absorbed more readily [35].

Flavonoid intake is negatively correlated with the incidence of several chronic diseases including cardiovascular diseases, type II diabetes, neurodegenerative diseases, and cancers $[12,36]$. As described by Hollman, flavonoid glucosides are generally the only glycosides that can be absorbed from the small intestine. After absorption from the small intestine, flavonoids are conjugated with glucuronic acid, sulphate, or O-methylation and, as a result, no free flavonoid aglycones can be found in plasma or urine, except for catechins. Flavonoids that cannot be absorbed from the small intestine will be degraded in the colon by microorganisms, which will break down the flavonoid ring structure. The resulting phenolic acids can be absorbed and have been measured in plasma and urine [37].

Phenolic acids are extensively studied due to their bioactive properties and there is evidence of their role in disease prevention [38]. Like other phenolic compounds they are extensively metabolised and circulate in the organism as glucuronated, sulphated, and methylated metabolites, displaying different bioactivities compared to their precursors [38]. Likewise, hydroxycinnamic acids, naturally occurring anti-inflammatory bioactive compounds, are also extensively metabolised [39].

A versatile range of phytochemicals that were tentatively identified during this study are reported to be beneficial for human health. F. ulmaria or extracts thereof may therefore be considered as a promising source for future functional ingredients. However, all the findings on the extensive metabolisation of phenolic constituents urge the need for a shift of (poly)phenol research towards intestinal, colonic, and hepatic metabolites as the principal bioactives $[12,38,39]$. Although the exact structure of the phenolic constituents identified during this study (e.g., the nature of the sugars and the interglycosidic linkages of glycosides) often cannot be established with LC-amMS without reference standards, these linkages are frequently broken during metabolisation. Comprehensive and fast LC-amMS profiling should be combined with pharmacological evaluation of plant extracts before and after metabolisation to enable the identification of potential pro-drugs that otherwise may be overlooked.

Next to phenolic compounds, only few and often low abundant non-phenolic phytochemicals were detected in Filipendulae Ulmariae Herba. Several triterpenes were tentatively identified. Ursolic and pomolic acid were previously identified by Halkes as being the major compounds in the roots of $F$. ulmaria inhibiting T-lymphocyte proliferation [11]. Ursolic acid belongs to the pentacyclic triterpenes class of compounds, which is widely distributed in the plant kingdom and is primarily responsible for the anti-inflammatory activity of a variety of medicinal plants [40]. The chlorophyll derivatives, phytosterols, and carotenoids detected in low abundance during this study are ubiquitously present in nature and no relevant contribution to the specific pharmacological activity of $F$. ulmaria is expected.

In conclusion, the versatile phytochemical composition of Filipendulae Ulmariae Herba was comprehensively characterised for the first time with two complementary generic UHPLC-PDAamMS methods. Selective ion fragmentation with a hybrid quadrupole-orbital trap MS analyser proved to be a valuable tool for identification of unknown compounds in a complex matrix such as F. ulmaria. A total of 119 compounds (not including isomers) were tentatively identified, of which 69 compounds have never been reported in $F$. ulmaria before. Several metabolic precursors of salicylic acid, the in vivo metabolite responsible for part of the pharmacological activity of F. ulmaria, were detected. However, next to salicylates, a rich diversity of phenolic constituents (including various oligomeric phenols) was tentatively identified. Only few and often low abundant non-phenolic phytochemicals were detected. Various detected phytochemicals are reported to 
be beneficial for human health, however, in view of the phenolic nature of the main constituents, extensive metabolisation after oral intake before absorption can be expected. This urges the need for research towards the identification and activity of the intestinal, colonic, and hepatic metabolites of F. ulmaria.

\section{Materials and Methods}

$\nabla$

\section{Chemicals}

UHPLC-grade methanol, acetonitrile, and ethyl acetate were purchased from Biosolve. Ultrapure water with a resistivity of $18.2 \times \mathrm{M} \Omega \times \mathrm{cm}$ at $25^{\circ} \mathrm{C}$ was generated with a Millipore system. Dichloromethane for gas chromatography, $n$-hexane for gas chromatography, acetone for gas chromatography, and sodium hydrogen carbonate were purchased from Merck. Formic acid, acetic acid, ammonium formate, ammonium acetate, (D-Ala ${ }^{2}$ )-leucine encephalin, butylated hydroxytoluene (BHT), and sand (quartz) were supplied by Sigma-Aldrich. Commercially available mixtures to calibrate the mass spectrometer, i.e., MSCAL5-1EA (caffeine, tetrapeptide "Met-Arg-Phe-Ala", Ultramark) for the positive ion mode and MSCAL6-1EA (sodium dodecylsulfate, taurocholic acid sodium salt, Ultramark) for the negative ion mode, were purchased from Supelco.

The following analytical standards were purchased from Phytolab: apigenin, luteolin, isorhamnetin, kaempferol, kaempferol-3O-glucoside (astragalin), quercetin, quercetin-3-O-glucoside (isoquercitrin), quercetin-3-0-galactoside (hyperin), quercetin3-O-rutinoside (rutin), quercetin-3-O-arabinoside (avicularin), quercetin-3-0-rhamnoside (quercitrin), galangin, phloretin, naringenin, (+)-catechin, (-)-epicatechin, (+)-dihydrokaempferol [(+)-aromadendrin], cyanidin-3-O-glucoside chloride (kuromanin chloride), cyanidin-3-O-rutinoside chloride (keracyanin chloride), procyanidin B2, ellagic acid, and eriodictyol. Analytical standards of salicylic acid, protocatechuic acid, gallic acid, p-coumaric acid, caffeic acid, chlorogenic acid, $\beta$-carotene, stigmasterol, $\beta$-sitosterol, miquelianin (quercetin 3-O-glucuronide), tannic acid, $\gamma$-tocopherol, $\alpha$-tocopherol, and quinic acid were obtained from Sigma-Aldrich. Lutein and violaxanthin were purchased from Carotenature.

Filipendulae Ulmariae Herba (batch number 19969) was bought from Tilman SA. A certificate of analysis describing the identification of Filipendulae Ulmariae Herba is in accordance with the specifications of organoleptic, microscopic, macroscopic, chromatographic, and steam distillation tests described in the European Pharmacopoeia and was provided by Tilman SA [41].

\section{Preparation of standard solutions}

Standard stock solutions for the phenolic analytes were prepared at a concentration of $1 \mathrm{mg} / \mathrm{mL}$ in UHPLC-grade methanol for each analyte separately and stored in the dark at $4{ }^{\circ} \mathrm{C}$. Dilutions of these solutions were prepared in $60: 40(\mathrm{v}: \mathrm{v})$ methanol:40 mM ammonium formate buffer (aqueous).

Standard stock solutions and working solutions for the non-phenolic analytes were prepared for each analyte separately at a concentration of approximately $200 \mu \mathrm{g} / \mathrm{mL}$. The stock solutions of phytosterols and lipid-soluble vitamins were prepared in methanol $+0.1 \%$ BHT. Stock solutions of carotenoids were prepared in dichloromethane $+0.1 \%$ BHT. Standard stock and working solutions were stored at $-25^{\circ} \mathrm{C}$ in the dark under an inert atmosphere (nitrogen). Dilutions of these solutions were prepared in dichloromethane $+0.1 \%$ BHT for analysis.

\section{Sample preparation}

The sample material was ground prior to extraction with an MF 10 basic Microfine grinder drive (IKA-Werke GmbH \& Co. KG) using a sieve mesh size of $0.5 \mathrm{~mm}$. Two generic sample preparation protocols were developed previously with the aim to be complementary in terms of polarity of extracted compounds $[20,21]$. During this study, these two complementary extraction protocols were combined and used in parallel for the first time. The combination of the two extraction methods enables the full range of phytochemical constituents to be extracted. All sample extractions were performed in triplicate.

\section{Extraction of moderately polar phytochemicals}

An extraction protocol previously developed by De Paepe et al. was applied for the extraction of moderately polar phytochemicals [20]. Briefly, $1 \mathrm{~g}$ of Filipendulae Ulmaria Herba was extracted with methanol:40 $\mathrm{mM}$ ammonium formate buffer (aqueous) $(20: 80, v: v)$ in a first step and $40 \mathrm{mM}$ ammonium formate in methanol in a second step. Each extraction was performed by ultrasound-assisted solid-liquid extraction with $10 \mathrm{~mL}$ of the appropriate solvent by using a 2200 R-4 Ultrasonic sonicator (40 kHz, $100 \mathrm{~W}$ ) (Branson Ultrasonic Corporation) for $60 \mathrm{~min}$ at room temperature. After 30 min of extraction, the solutions were vortex mixed (IKA MS2 Minishaker, IKAWerke GmbH \& Co. KG). During sonication, the temperature was kept below $40^{\circ} \mathrm{C}$. The samples were subsequently centrifuged at $3000 \mathrm{rpm}$ (approximately $1450 \mathrm{~g}$ ) using an Allegra ${ }^{\mathrm{TM}}$ Centrifuge (Beckman Coulter Inc.). Following the two consecutive extraction cycles, the supernatants were combined, diluted 5 times, and stored at $4{ }^{\circ} \mathrm{C}$ until analysis.

\section{Extraction of apolar phytochemicals}

A method previously developed by Bijttebier et al. was used for the extraction of a wide array of apolar phytochemicals [21]. Approximately $1 \mathrm{~g}$ of Filipendulae Ulmaria Herba was spiked with trans- $\beta$-Apo-8'-carotenal (internal standard). The sample was subsequently mixed with approximately $1 \mathrm{~g}$ of sodium hydrogen carbonate and sand. Ultrapure water was added until the sample was hydrated (approximately $3 \mathrm{~mL}$ ) and was let to rest in the dark under $\mathrm{N}_{2}$ for 30 min to allow swelling of the matrix for better analyte extraction. Afterwards, the mixture was homogenized with sand and loaded into a 33-mL Accelerated Solvent Extraction (ASE) cell (Thermo Fisher Scientific). The mixture was extracted 3 times with $70: 30$ acetone: methanol $+0.1 \%$ BHT (v:v) at $40{ }^{\circ} \mathrm{C}$ and $1050 \mathrm{psi}$. The three extracts were combined in a separating funnel and $100 \mathrm{~mL}$ of $10 \% \mathrm{NaCl}$ (aqueous) and $15 \mathrm{~mL}$ of hexane was added. The hexane phase was transferred to a recipient after vigorous shaking and the polar phase was extracted twice more with $15 \mathrm{~mL}$ hexane. The combined hexane fractions were evaporated to dryness, dissolved in $10 \mathrm{~mL}$ dichloromethane $+0.1 \%$ BHT and stored in the dark under nitrogen at $-25^{\circ} \mathrm{C}$ until analysis.

\section{Instrumental analysis}

Analogous to the complementary extraction methods, two generic LC-PDA-amMS methods were developed previously on an orbitrap MS (Exactive ${ }^{\mathrm{TM}}$; Thermo Fisher Scientific) with the aim to be complementary in the polarity range of analysed compounds $[20,21]$. These methods were used for the first time in parallel during the current study to enable the characterisation of the full range of phytochemical constituents. Moreover, the analytical methods were further improved by using a hybrid quadrupole-orbital trap MS analyser (Q Exactive ${ }^{\mathrm{TM}}$; Thermo Fisher Sci- 
entific), thereby enabling selective ion fragmentation to obtain clean compound spectra.

Because of the limitations in acquisition speed of the orbitrap detector, the sample extracts had to be analysed 3 times to gather the desired spectral information. A first analysis was performed by switching polarities (positive and negative) during ionisation to comprehensively detect compounds in both polarities in a single run. A second and third analysis was performed to selectively fragment the generated ions with HCD in the HCD cell before detection with the orbitrap mass analyser. The precursor ions generated by ionisation were selected for fragmentation based on their abundances during ddMS2. These consecutive ddMS ${ }^{2}$ experiments were performed in positive and negative ionisation modes, respectively.

In cases where ddMS ${ }^{2}$ did not allow for the full characterisation of the compound substructures because of insufficient product ions, in-source CID fragmentation was used in combination with HCD fragmentation to obtain pseudo MS ${ }^{3}$ spectra.

\section{Analysis of moderately polar phytochemicals}

The extracts containing moderately polar compounds were analysed with methodology adapted from De Paepe et al. [20]. For analysis, $5 \mu \mathrm{L}$ of extract were injected with a CTC PAL ${ }^{\mathrm{TM}}$ autosampler (CTC Analytics) on a Waters Acquity UPLC BEH SHIELD RP18 column ( $3.0 \mathrm{~mm} \times 150 \mathrm{~mm}, 1.7 \mu \mathrm{m}$; Waters) and thermostatically $\left(40^{\circ} \mathrm{C}\right)$ eluted with an Accela ${ }^{\mathrm{TM}}$ quaternary solvent manager and a "Hot Pocket" column oven (Thermo Fisher Scientific). The mobile phase solvents consisted of water $+0.1 \%$ formic acid $(\mathrm{A})$ and acetonitrile $+0.1 \%$ formic acid $(B)$, and the gradient was set as follows (min/A\%): 0.0/100, 9.91/74, 18.51/35, 18.76/0, 20.76/0, 20.88/100, 23.00/100. For detection, an amMS (Q Exactive ${ }^{\mathrm{TM}}$; Thermo Fisher Scientific) was used with HESI. During the first analysis, full scan data were acquired using polarity switching with a $m / z$ range of $120-1800$ and resolving power set at 70000 at FWHM. The spray voltage was set at $\pm 2.5 \mathrm{kV}$, sheath gas and auxiliary gas at 47 and 15 (adimensional), respectively, and capillary temperature at $350^{\circ} \mathrm{C}$. Data were also recorded using ddMS 2 in the positive and negative ionisation modes (one analysis per mode) to obtain additional structural information (resolving power set at 17500 FWHM, stepped collision energy 10, 30, $50 \mathrm{~V}$, isolation window: $4 \mathrm{~m} / z$, top 10 of most abundant ions selected for fragmentation). The PDA detector was set to scan from 190 to $800 \mathrm{~nm}$ during all analyses.

\section{Analysis of apolar phytochemicals}

The extracts containing apolar compounds were analysed with methodology adapted from Bijttebier et al. [21]. For analysis, $1.25 \mu \mathrm{L}$ of extract was injected on a Waters Acquity UPLC HSS C18 SB column $(2.1 \mathrm{~mm} \times 100 \mathrm{~mm}, 1.8 \mu \mathrm{m}$; Waters $)$ and thermostatically $\left(35^{\circ} \mathrm{C}\right)$ eluted. The mobile phase solvents consisted of $50: 22.5: 22.5: 5(\mathrm{v}: \mathrm{v}: \mathrm{v}: \mathrm{v})$ water $+5 \mathrm{mM}$ ammonium acetate: methanol:acetonitrile: ethyl acetate (A) and 50:50 (v:v) acetonitrile: ethyl acetate $(B)$, and the gradient was set as follows (min/A\%): 0.0/90, 0.1/90, 0.8/70, 20.0/9, 20.1/0, 20.4/0, 20.5/90, 23.0/90. Atmospheric pressure chemical ionisation (APCI) was used as an MS ionisation technique. During the first analysis, full scan data were acquired using polarity switching with a $\mathrm{m} / \mathrm{z}$ range of 90-1400 and resolving power set at 70000 at FWHM. The corona discharge current was set at $\pm 5 \mu \mathrm{A}$, the vaporizer and capillary temperatures were set at $450^{\circ} \mathrm{C}$ for both the positive and negative APCI. Lock mass correction with $\left(\mathrm{D}-\mathrm{Ala}^{2}\right)$-leucin enkephalin was applied. Data were also recorded using ddMS ${ }^{2}$ in the positive and negative ionisation modes (one analysis per mode) to obtain additional structural information (resolving power set at 17500 FWHM, stepped collision energy 10, 30, $50 \mathrm{~V}$, isolation window: $4 \mathrm{~m} / z$, top 10 of most abundant ions selected for fragmentation). The PDA detector was set to scan from 190 to $800 \mathrm{~nm}$ during all analyses.

\section{Acknowledgements \\ $\nabla$}

We thank the Flemish Fund for Scientific Research (FWO) for supporting this research (project 12M8315N).

\section{Conflict of Interest}

$\nabla$

There are no conflicts of interest to disclose.

\section{References}

1 European Medicines Agency. Assessment report on Filipendula ulmaria (L.) Maxim., herba and Filipendula ulmaria (L.) Maxim., flos. Available at http://www.ema.europa.eu/ema. Accessed December 3, 2015

2 Harbourne N, Jacquier JC, O'Riordan D. Optimisation of the aqueous extraction conditions of phenols from meadowsweet (Filipendula ulmaria L.) for incorporation into beverages. Food Chem 2009; 116: 722727

3 Okuda T, Yoshida T, Hatano T, Iwasaki M, Kubo M, Orime T, Yoshizaki M, Naruhashi N. Hydrolysable tannins as chemotaxonomic markers in the Rosaceae. Phytochem 1992; 31: 3091-3096

4 Pemp E, Reznicek G, Krenn L. Fast quantification of flavonoids in Filipendulae Ulmariae Flos by HPLC/ESI-MS using a nonporous stationary phase. J Anal Chem 2007; 62: 669-673

5 Papp I, Simándi B, Blazics B, Alberti Á, Héthelyi É, Szöke É, Kéry Á. Monitoring volatile and non-volatile salicylates in Filipendula ulmaria by different chromatographic techniques. Chromatogr Suppl 2008; 68: S125-S129

6 Fecka I. Qualitative and quantitative determination of hydrolysable tannins and other polyphenols in herbal products from meadowsweet and dog rose. Phytochem Anal 2009; 20: 177-190

7 Shilova IV, Semenov AA, Suslov NI, Korotkova EI, Vtorushina AN, Belyako$v a \mathrm{VV}$. Chemical composition and biological activity of a fraction of meadowsweet extract. Pharm Chem J 2009; 43: 185-190

8 Barros L, Alves CT, Dueñas M, Silva S, Oliveira R, Carvalho AM, Henriques $M$, Santos-Buelga C, Ferreira ICFR. Characterization of phenolic compounds in wild medicinal flowers from Portugal by HPLC-DAD-ESI/MS and evaluation of antifungal properties. Ind Crops Prod 2013; 44: 104110

9 Olennikov DN, Kruglova MY. A new quercetin glycoside and other phenolic compounds from the genus Filipendula. Chem Nat Compd 2013; 49: 610-616

10 Barros L, Cabrita L, Vilas Boas M, Carvalho AM, Ferreira ICFR. Chemical, biochemical and electrochemical assays to evaluate phytochemicals and antioxidant activity of wild plants. Food Chem 2011; 127: 16001608

11 Halkes SBA. Filipendula ulmaria - a study on the immunomodulary activity of extracts and constituents [dissertation]. Utrecht: Universiteit Utrecht; 1998

12 Del Rio D, Rodriguez-Mateos A, Spencer JPE, Tognolini M, Borges G, Crozier A. Dietary (poly)phenolics in human health: structures, bioavailability, and evidence of protective effects against chronic diseases. Antioxid Redox Signal 2013; 18: 1818-1892

13 Butterweck $V$, Nahrstedt $A$. What is the best strategy for preclinical testing of botanicals? A critical perspective. Planta Med 2012; 78: 747-754

14 Mills S, Bone K. Principles and practice of phytotherapy. Modern herbal medicine. Edinburgh: Churchill Livingstone; 2000

15 Ojanperä I, Kolmonen M, Pelander A. Current use of high-resolution mass spectrometry in drug screening relevant to clinical and forensic toxicology and doping control. Anal Bioanal Chem 2012; 403: 12031220 
16 Christensen $L P$, Brandt $K$. Bioactive polyacetylenes in food plants of the Apiaceae family: occurrence, bioactivity and analysis. J Pharm Biomed Anal 2006; 41: 683-693

17 Xu YJ, Foubert K, Dhooghe L, Lemière F, Maregesi S, Coleman CM, Zou Y, Ferreira D, Apers S, Pieters $L$. Rapid isolation and identification of minor natural products by LC-MS, LC-SPE-NMR and ECD: isoflavanones, biflavanones and bisdihydrocoumarins from Ormocarpum kirkii. Phytochemistry 2012; 79: 121-128

18 Ibáñez C, García-Cañas V, Valdés A, Simó C. Novel MS-based approaches and applications in food metabolomics. Trends Anal Chem 2013; 52: 100-111

19 Kind T, Fiehn 0 . Metabolomic database annotations via query of elemental compositions: mass accuracy is insufficient even at less than 1 ppm. BMC Bioinformatics 2006; 7: 234

20 De Paepe D, Servaes K, Noten B, Diels L, De Loose M, Van Droogenbroeck $B$, Voorspoels $S$. An improved mass spectrometric method for identification and quantification of phenolic compounds in apple fruits. Food Chem 2013; 136: 368-375

21 Bijttebier S, Zhani K, D'Hondt E, Noten B, Hermans N, Apers S, Voorspoels $S$. Generic characterization of apolar metabolites in red chili peppers (Capsicum frutescens L.) by Orbitrap mass spectrometry. J Agric Food Chem 2014; 62: 4812-4831

22 Cuyckens F, Claeys M. Mass spectrometry in the structural analysis of flavonoids. J Mass Spectrom 2004; 39: 1-15

23 Sun J, Liang F, Bin Y, Li P, Duan C. Screening non-colored phenolics in red wines using liquid chromatography/ultraviolet and mass spectrometry/mass spectrometry libraries. Molecules 2007; 12: 679-693

24 Blazics B. Analysis of medicinal plant phenoloids by coupled tandem mass spectrometry [dissertation]. Budapest, Hungary: Semmelweis University; 2010

25 Krasnov EA, Raldugin VA, Shilova IV, Avdeeva EY. Phenolic compounds from Filipendula ulmaria. Chem Nat Compd 2006; 42: 148-151

26 Regueiro J, Sánchez-González C, Vallverdú-Queralt A, Simal-Gándara J, Lamuela-Raventós R, Izquierdo-Pulido M. Comprehensive identification of walnut polyphenols by liquid chromatography coupled to linear ion trap-Orbitrap mass spectrometry. Food Chem 2014; 152: 340-348

27 Mingshu L, Kai Y, Qiang H, Dongying J. Biodegradation of gallotannins and ellagitannins. J Basic Microbiol 2006; 46: 68-84

28 Niemetz R, Schilling G, Gross GG. Ellagitannin biosynthesis: oxidation of pentagalloylglucose to tellimagrandin II by an enzyme from Tellima grandiflora leaves. Chem Commun 2001; 1: 35-36
29 Landete JM. Ellagitannins, ellagic acid and their derived metabolites: A review about source, metabolism, functions and health. Food Res Int 2011; 44: 1150-1160

30 Li YJ, Wei HL, Qi LW, Chen J, Ren MT, Li P. Characterization and identification of saponins in Achyranthes bidentata by rapid-resolution liquid chromatography with electrospray ionization quadrupole time-offlight tandem mass spectrometry. Rapid Commun Mass Spectrom 2010; 24: 2975-2985

31 Bale NJ, Airs RL, Llewellyn CA. Type I and Type II chlorophyll- $a$ transformation products associated with algal senescence. Org Geochem 2011; 42: 451-464

32 Choy YY, Waterhouse AL. Proanthocyanidin metabolism, a mini review. Nutr Aging 2014; 2: 111-116

33 Shukla M, Gupta K, Rasheed Z, Khan KA, Haqqi TM. Consumption of hydrolyzable tannins-rich pomegranate extract suppresses inflammation and joint damage in rheumatoid arthritis. Nutr 2008; 24: 733-743

34 Rasmussen SE, Frederiksen H, Krogholm KS, Poulsen L. Dietary proanthocyanidins: occurrence, dietary intake, bioavailability, and protection against cardiovascular disease. Mol Nutr Food Res 2005; 49: 159-174

35 Rzeppa S, Bittner K, Döll S, Dänicke S, Humpf HU. Urinary excretion and metabolism of procyanidins in pigs. Mol Nutr Food Res 2012; 56: 653665

36 Lu MF, Xiao ZT, Zhang HY. Where do health benefits of flavonoids come from? Insights from flavonoid targets and their evolutionary history. Biochem Biophys Res Commun 2013; 434: 701-704

37 Hollman PCH. Absorption, bioavailability, and metabolism of flavonoids. Pharm Biol 2004; 42: 74-83

38 Heleno SA, Martins A, Queiroz MJRP, Ferreira ICFR. Bioactivity of phenolic acids: metabolites versus parent compounds: a review. Food Chem 2015; 173: 501-513

39 Farrell T, Poquet L, Dionisi F, Barron D, Williamson G. Characterization of hydroxycinnamic acid glucuronide and sulfate conjugates by HPLCDAD-MS 2 : enhancing chromatographic quantification and application in Caco-2 cell metabolism. J Pharm Biomed Anal 2011; 55: 1245-1254

40 Alvarado HL, Abrego G, Garduño-Ramirez ML, Clares B, Calpena AC, García ML. Design and optimization of oleanolic/ursolic acid-loaded nanoplatforms for ocular anti-inflammatory applications. Nanomedicine 2015; 11: 521-530

41 European Pharmacopoeia, 8.0. London: 04/2013; 1868: 1316-1317

42 Wilkes $S$, Glasl $\mathrm{H}$. Isolation, characterization, and systematic significance of 2-pyrone-4,6-dicarboxylic acid in Rosaceae. Phytochemistry 2001; 58: 441-449 